\title{
The Higgs and top mass coincidence problem
}

\author{
E. Torrente-Lujan ${ }^{1, a}$ \\ ${ }^{1}$ IFT, Dept. of Physics, U. Murcia, Murcia, SPAIN.
}

\begin{abstract}
.
On the light of the recent LHC boson discovery, we present a phenomenological evaluation of the ratio $\rho_{t}=m_{Z} m_{t} / m_{H}^{2}$, from the LHC combined $m_{H}$ value, we get $((1 \sigma))$

$$
\rho_{t}^{(e x p)}=0.9956 \pm 0.0081 .
$$

This value is close to one with a precision of the order $\sim 1 \%$. Similarly we evaluate the ratio $\rho_{W t}=\left(m_{W}+m_{t}\right) /\left(2 m_{H}\right)$. From the up-to-date mass values we get $\rho_{W t}^{(e x p)}=$ $1.0066 \pm 0.0035(1 \sigma)$. The Higgs mass is numerically close (at the $1 \%$ level) to the $m_{H} \sim\left(m_{W}+m_{t}\right) / 2$. From these relations we can write any two mass ratios as a function of, exclusively, the Weinberg angle (with a precision of the order of $1 \%$ or better):

$$
\frac{m_{i}}{m_{j}} \simeq f_{i j}\left(\theta_{W}\right), \quad i, j=W, Z, H, t .
$$

For example: $m_{H} / m_{Z} \simeq 1+\sqrt{2} s_{\theta_{W} / 2}^{2}, m_{H} / m_{t} c_{\theta_{W}} \simeq 1-\sqrt{2} s_{\theta_{W} / 2}^{2}$. In the limit $\cos \theta_{W} \rightarrow 1$ all the masses would become equal $m_{Z}=m_{W}=m_{t}=m_{H}$. It is tempting to think that such a value, it is not a mere coincidence but, on naturalness grounds, a signal of some more deeper symmetry. In a model independent way, $\rho_{t}$ can be viewed as the ratio of the highest massive representatives of the spin $(0,1 / 2,1) \mathrm{SM}$ and, to a very good precision the LHC evidence tell us that $m_{s=1} m_{s=1 / 2} / m_{s=0}^{2} \simeq 1$. Somehow the "lowest" scalar particle mass is the geometric mean of the highest spin 1, 1/2 masses.

We review the theoretical situation of this ratio in the SM and beyond. In the SM these relations are rather stable under RGE pointing out to some underlying UV symmetry. In the SM such a ratio hints for a non-casual relation of the type $\lambda \simeq \kappa\left(g^{2}+g^{\prime 2}\right)$ with $\kappa \simeq 1+$ $o\left(g / g_{t}\right)$. Moreover the existence of relations $m_{i} / m_{j} \simeq f_{i j}\left(\theta_{W}\right)$ could be interpreted as a hint for a role of the $S U(2)_{c}$ custodial symmetry, together with other unknown mechanism. Without a symmetry at hand to explain then in the SM, it arises a Higgs mass coincidence problem, why the ratios $\rho_{t}, \rho_{W t}$ are so close to one, can we find a mechanism that naturally gives $m_{H}^{2}=m_{Z} m_{t}, 2 m_{H}=m_{W}+m_{t}$ ?

PACS:14.80.Bn,14.80.Cp.
\end{abstract}

\section{The ratio $\rho_{t}=m_{Z} m_{t} / m_{H}$}

The problem of the mass of elementary particles has two independent aspects in particle physics. The first, how mass arises, it is answered in the SM by the Higgs mechanism. For composite particles,

\footnotetext{
a e-mail: etl@um.es
} 
i.e. hadrons, the dynamical generation of mass is indeed a dominant mechanism. The second aspect is why different elementary particles have their specific masses. Unless electromagnetic charge, there is no any, exact or approximate, known relation, structure or hierarchy among the masses of the SM elementary particles.

Evidence in favour of the existence of a boson with spin $s=0$ and properties compatible with those of a SM Higgs particle (production cross sections, mass-proportional couplings) has been presented by the LHC experiments $[3,4]$.

The aim of this work ${ }^{1}$ is to present some detailed phenomenological estimations of some ratios involving the experimental masses of the Higgs boson, the vector bosons and the top quark, the derivation from them of some model independent expressions and detailed study of them in the framework of the SM. In the light of the recent results from the LHC coming from the experiments ATLAS and CMS, the parameter defined by the relation

$$
\rho_{t}=\frac{m_{Z} m_{t}}{m_{H}^{2}}
$$

where $m_{Z}, m_{t}$ are the masses of the $Z^{0}$ gauge boson and the top quark and $m_{H}$ is the Higgs mass has become experimentally measurable. We estimate its current value to be

$$
\rho_{t}^{(\exp )}=0.9956 \pm 0.0081
$$

where we have used the current values for [2]

$$
\begin{aligned}
m_{Z} & =91.1876 \pm 0.0021 \mathrm{GeV} / \mathrm{c}^{2} \\
m_{t} & =173.07 \pm 0.52 \pm 0.72 \mathrm{GeV} / \mathrm{c}^{2}
\end{aligned}
$$

and the combined value of the boson masses presented by ATLAS and CMS [3, 4],

$$
m_{H}=125.9 \pm 0.4 \pm 0.4 \mathrm{GeV} / \mathrm{c}^{2} .
$$

The combined value of the boson mass is obtained by standard statistic techniques, we neglect correlations among the systematic component of the errors. The value (3) is obtained by a MC simulation. First, a distribution of the quotient is obtained by generating Gaussian ensembles of the individual masses. Second, symmetric gaussian fit, fig.(1), is performed to the central part of this (close to symmetric) distribution avoiding the non-gaussian tail. The central value and $1 \sigma$ errors appearing in (3) are extracted from this fitted gaussian. If the individual values for each ot the experiments are used instead (using a similar statistical procedure), we get (see fig.(1)(up))

$$
\begin{array}{lll}
\rho_{t}^{(\text {exp })}=0.9940 \pm 0.0102 & \left(m_{h, A T L A S}\right), \\
\rho_{t}^{(\text {exp })}=0.9990 \pm 0.0085 & \left(m_{h, C M S}\right)
\end{array}
$$

for boson masses respectively

$$
m_{H}=125.8 \pm 0.4 \pm 0.4 \mathrm{GeV} / \mathrm{c}^{2}
$$

and

$$
m_{H}=126.0 \pm 0.4 \pm 0.4 \mathrm{GeV} / \mathrm{c}^{2} .
$$

The conclusion is that the experimental value of the ratio $\rho_{t}$ is close to one with a precision of the order or less than $1 \%$. This precision is not far from the precision at which the well known ratio

$$
\rho=m_{W}^{2} / m_{Z}^{2} \cos ^{2} \theta_{W}
$$

\footnotetext{
${ }^{1}$ This presentation is based in [1]
} 
is presentely measured, $\rho=1.0008 \pm 0.001$ [2] with $\theta_{W}$ the Weinberg angle and $m_{W}$ the charged electroweak gauge boson mass. The closeness of this parameter $\rho_{t}$ to one might be merely a coincidence which will dissapear with any new measurement or might be not.

Note that the ratio would be exactly one for a boson mass (and nominal $m_{Z}, m_{t}$ PDG masses) of

$$
m_{H}\left(\rho_{t}=1\right) \simeq 125.6 \mathrm{GeV} / \mathrm{c}^{2},
$$

a value somewhere inbetween of the 125 - 126 range of values currently measured by LHC and just on the borderline of the SM vacuum stability limits [12].

The ratio $\rho_{t}$ would be still close to one, with a precision of $5 \%$, if the Higgs mass appear finally anywhere in the range $m_{H}=123-129 \mathrm{GeV} / \mathrm{c}^{2}$. If we vary the top mass in the range $m_{t} \sim 170-175$ $\mathrm{GeV} / \mathrm{c}^{2}$ similar results are obtained.

Similar ratios involving other fermion masses instead of the top mass could be obviously defined, for example including all the fermions we could define $\rho_{\Sigma}$ as

$$
\rho_{\Sigma}=\frac{m_{Z} m_{\Sigma}}{m_{H}^{2}},
$$

with

$$
m_{\Sigma}^{2}=\sum_{f} m_{f}^{2}
$$

or including the third family quark doublet $\left(m_{Q}^{2}=m_{t}^{2}+m_{b}^{2}\right)$ we could define the ratio

$$
\begin{aligned}
\rho_{T} & \equiv \frac{m_{Z} m_{Q}}{m_{H}^{2}} \\
& \simeq \rho_{t}\left(1+2\left(\frac{m_{b}}{m_{t}}\right)^{2}\right)
\end{aligned}
$$

In any case, any of these or similar ratios are deviated from $\rho_{t}$ by a very moderate quantity $\left(m_{b} / m_{t}\right)^{2} \simeq$ $10^{-3}$.

It is also interesting to consider an alternative way to express the closeness of the ratio $\rho_{t}$ to one. If we consider the individual mass rations $m_{Z} / m_{H}, m_{H} / m_{t}$, their current experimental values are ${ }^{2}$.

$$
\begin{aligned}
& \frac{m_{Z}}{m_{H}}=0.725 \pm 0.003 \\
& \frac{m_{H}}{m_{t}}=0.727 \pm 0.005
\end{aligned}
$$

where we have taken the LHC combined value of $m_{H}$. and PDG $m_{Z}, m_{t}$ masses. Both ratios are the same at the level of $1 \%$ (and totally compatible at even higher precision according to present error bars). Very similar results are obtained if we use any of the ATLAS or CMS individual measurements

Similarly to $\rho_{t}$ we define now another ratio of masses involving the Higgs, vector bosons and top quarks, whose experimental value is also seen to be close to one. Let us take

$$
\rho_{W t}=\frac{m_{W}+m_{t}}{2 m_{H}}
$$

\footnotetext{
2 "God" or "golden" particle?. The difference between any of the $m_{H} / m_{Z}, m_{Z} / m_{H}$ ratios and the Golden Ratio $(\sqrt{ } 5+1) / 2$ is a "mere" $15 \%$. Equality would be exact if $m_{t}=m_{H}+m_{Z}$
} 

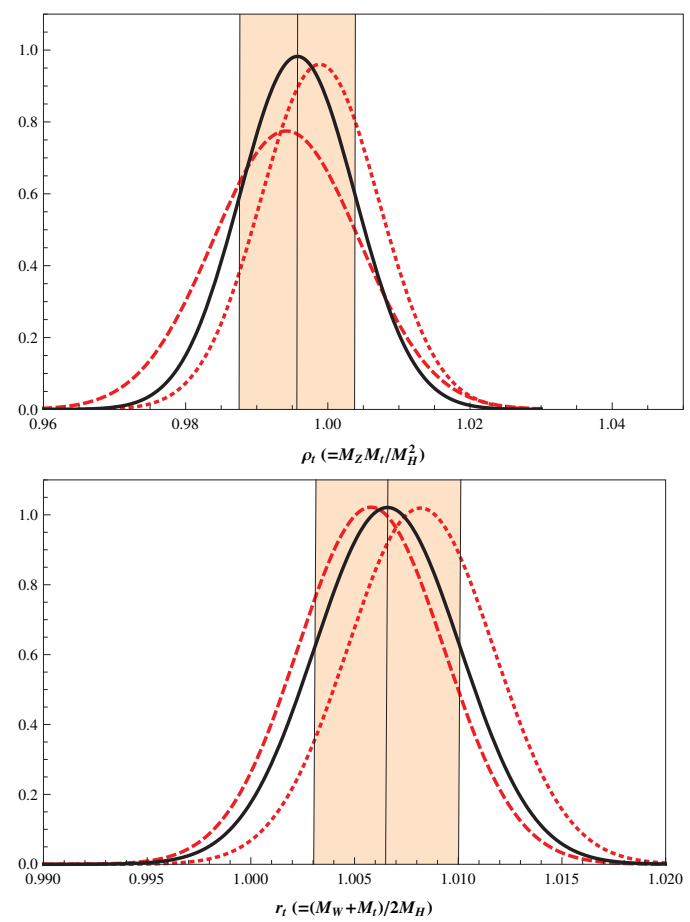

Figure 1. MC generated Likelihood distributions for each of the quantities $\rho_{t}$ (up) and $\rho_{W t}$ (down). The curves are in arbitrary units with normalized areas. The curves correspond to the global averaged Higgs mass (continuos) and individual ATLAS and CMS values (dashed and dotted lines). The shaded area is the $1 \sigma$ allowed region for each of the parameters.

where $m_{W}$ is the mass of the $W$ boson. We estimate the current value of this ratio (using a similar MC technique as explained above, see fig.(1)(down)) to be

$$
\rho_{W t}^{(e x p)}=1.0066 \pm 0.0035
$$

where we have used the current value for $M_{W}[2]$

$$
M_{W}=80.385 \pm 0.015 \mathrm{GeV} / \mathrm{c}^{2}
$$

and the combined value for the Higgs mass, Eq.(6). If the individual values for each of the experiments are used instead, we get

$$
\begin{array}{ll}
\rho_{W t}^{(e x p)}=1.0082 \pm 0.0036 & \left(m_{h, A T L A S}\right), \\
\rho_{W t}^{(e x p)}=1.0056 \pm 0.0036 & \left(m_{h, C M S}\right) .
\end{array}
$$

The experimental value of the ratio $\rho_{W t}$ is close to one with a precision of the order of $1 \%$. Nevertheless the favoured value is around $2 \sigma$ from being exactly one. The Higgs mass is numerically close to the aritmethic average of the charged boson and top masses $m_{H} \sim\left(m_{W}+m_{t}\right) / 2$. The ratios $\rho_{t}$ and $\rho_{W t}$ are a priori independent. There is no reason why the value of one of them should be close to one even if the other happens to be close to one. 
The relations $\rho_{t} \simeq \rho_{W t} \simeq 1$ imply that any two of the quantities $m_{H}, m_{W}, m_{Z}, m_{t}$ can be written in terms of the other two. Taking into account also the relation $\rho \simeq 1$ we can write any two mass ratios as a function of, exclusively, the Weinberg angle (with a precision of the order of $1 \%$ or better):

$$
\frac{m_{i}}{m_{j}} \simeq f_{i j}\left(\theta_{W}\right), \quad i, j=W, Z, H, t
$$

Examples of these relations are:

$$
\begin{aligned}
\frac{m_{W}}{m_{Z}} & \simeq \cos \theta_{W}, \\
\frac{m_{H}}{m_{Z}} & \simeq 1+\sqrt{2} \sin ^{2} \frac{\theta_{W}}{2}, \\
\frac{m_{H}}{m_{t}} \cos \theta_{W} & \simeq 1-\sqrt{2} \sin ^{2} \frac{\theta_{W}}{2} .
\end{aligned}
$$

In the limit $\cos \theta_{W} \rightarrow 1$ all the masses would become equal $m_{Z}=m_{W}=m_{t}=m_{H}$.

\section{In the SM}

The latest LHC measurements [3, 4], in particular the characteristics of the seen $H \rightarrow \gamma \gamma$ channel, point to a preferred discovery of a neutral boson of spin 0 . In a model independent way, thus the quantity $\rho_{t}$ can be viewed as the ratio of the highest massive representatives of the spin $(0,1 / 2,1)$ particles of the Standard Model and, to a very good precision the experimental evidence tell us that

$$
\rho_{t}^{(\exp )} \sim \frac{m_{s=1} m_{s=1 / 2}}{m_{s=0}^{2}} \simeq 1 .
$$

Somehow the mass of the "lowest" scalar particle mass is numerically the geometric mean of the highest spin 1 and spin $1 / 2$ masses.

Let us now assume that the new particle is a scalar Higgs boson. In the Standard Model (SM) with a Higgs sector consistent of one Higgs doublet $\Phi$ and scalar potential

$$
V_{S M}=\mu^{2} \Phi^{\dagger} \Phi+\lambda\left(\Phi^{\dagger} \Phi\right)^{2},
$$

all masses are induced by spontaneous symmetry breaking and are proportional to the Higgs vaccuum expectation $\left.<\phi_{0}\right\rangle=v / \sqrt{2}$ where

$$
v^{2}=-\frac{\mu^{2}}{\lambda}=\frac{1}{\sqrt{ } 2 G_{F}}=(246.218)^{2} \quad\left(\mathrm{GeV} / \mathrm{c}^{2}\right)^{2} .
$$

The tree level top, gauge and Higgs boson masses are given in terms of $v$ and their respective Yukawa couplings

$$
\begin{aligned}
m_{W} & =g \frac{v}{2}, \quad m_{Z}=\sqrt{g^{2}+g^{\prime 2}} \frac{v}{2} \\
m_{t} & =g_{t} \frac{v}{2} \\
m_{H}^{2} & =-2 \mu^{2}=2 \lambda v^{2} .
\end{aligned}
$$

Moreover we have $g^{\prime}=g \tan \theta_{W}$ or $\sqrt{g^{2}+g^{\prime 2}}=g / \cos \theta_{W}, G_{F} m_{W}^{2} / \sqrt{ } 2=g^{2} / 8$. 
In terms of these quantities the tree level mass ratio $\rho_{t}$ is simply given by

$$
\begin{aligned}
\rho_{t}^{O(S M)} & =\frac{m_{Z} m_{t}}{m_{H}^{2}}=\sqrt{g^{2}+g^{\prime 2}} \frac{v^{2} g_{t}}{4 \sqrt{2} v^{2} \lambda} \\
& =\frac{1}{4 \sqrt{ } 2} \frac{\sqrt{g^{2}+g^{\prime 2}} g_{t}}{\lambda} \\
& =\frac{1}{4 \sqrt{ } 2} \frac{g g_{t}}{\cos \theta_{W} \lambda} .
\end{aligned}
$$

Similarly, the tree level $\mathrm{SM} \rho_{W t}$ ratio is given by:

$$
\rho_{W t}^{0(S M)}=\frac{m_{W}+m_{t}}{2 m_{H}}=\frac{g+g_{t}}{4 \sqrt{ }(2 \lambda)} .
$$

Numerically, we estimate the top yukawa, quartic coupling and other related ratios as ( from measured masses, using tree level approximation, $1 \sigma$ errors ) :

$$
\begin{aligned}
g_{t}^{0,(\exp )} & =1.409 \pm 0.007, \\
\lambda^{0,(\exp )} & =0.130 \pm 0.001, \\
\left(\frac{\lambda}{g_{t}^{2}}\right)^{0,(\exp )} & =\frac{1}{8}\left(\frac{m_{H}}{m_{t}}\right)^{2}=(6.6 \pm 0.1) \times 10^{-2}, \\
\left(\frac{g^{2}+g^{\prime 2}}{\lambda}\right)^{0,(\exp )} & =8\left(\frac{m_{Z}}{m_{H}}\right)^{2}=4.21 \pm 0.03 .
\end{aligned}
$$

In the SM, the Higgs selfcoupling $\lambda$ is non-determined. However, assuming that both expressions $\rho_{t}, \rho_{W t} \simeq 1$ are not a coincidence, the relations (31) and (33) tell us that the scalar self-couplings, gauge couplings and Yukawa top couplings are related by two expressions. At tree level any two of the quantities $\lambda, g, g^{\prime}, g_{t}$ can be written in terms the two others using the expressions:

$$
\begin{aligned}
& \lambda \simeq c \sqrt{g^{2}+g^{\prime 2}} g_{t}, \\
& \lambda \simeq c^{2}\left(g+g_{t}\right)^{2}
\end{aligned}
$$

where $c$ is a numeric factor of order $\sim o(1)$. If we take into account only the first expression, the one related to the ratio $\rho_{t}$, we arrive to the relation between the quartic, gauge and top couplings

$$
\lambda \sim g g_{t} .
$$

Let us take now into account both expressions. For $g_{t}>>g$ the second equation becomes $\lambda \simeq c^{2} g_{t}^{2}$, inserting it in the first one we arrive to

$$
\lambda \simeq \kappa\left(g^{2}+g^{\prime 2}\right)
$$

with $\kappa \simeq 1+o\left(g / g_{t}\right)$.

The tree level relations $(31,33)$ are affected by SM quantum corrections. Including one loop corrections, the three level relations above should be replaced, in particular by (where $\mu_{0}$ the renormalization scale, $\mu_{0} \sim m_{Z}-m_{t}$ )

$$
\begin{aligned}
g_{t}\left(\mu_{0}\right) & =\frac{\sqrt{ } 2 m_{t}}{v}\left(1+\delta_{t}\left(\mu_{0}\right)\right), \\
\lambda\left(\mu_{0}\right) & =\frac{\sqrt{ } m_{H}^{2}}{2 v^{2}}\left(1+\delta_{\lambda}\left(\mu_{0}\right)\right),
\end{aligned}
$$


we consider negligible the running of the gauging couplings $g_{i}\left(\mu_{0}\right)$. The first order corrected ratio $\rho_{t}$ is then, using expresions $(42,43)$,

$$
\begin{aligned}
\rho_{t}^{S M} & =\frac{m_{Z} m_{t}}{m_{H}^{2}} \\
& =\frac{1}{4 \sqrt{ } 2} \frac{g g_{t}}{\cos \theta_{W} \lambda} \frac{1+\delta_{\lambda}}{1+\delta_{t}} \\
& \simeq \rho_{t}^{0}\left(1+\delta_{\lambda}-\delta_{t}\right) .
\end{aligned}
$$

The top yukawa $\delta_{t}$ can be written as $\delta_{t}=\delta_{t}^{Q C D}+\delta_{t}^{w}$. The corrections are ([5] and references therein), ignoring logarithm terms,

$$
\begin{aligned}
\delta_{\lambda} & =\frac{1}{16 \pi^{2}} c_{\lambda} \lambda, \\
\delta_{t}^{w} & =\frac{1}{16 \pi^{2}} \frac{c_{t}}{8} g_{t}^{2}, \\
\delta_{t}^{Q C D} & =\left(-1 /\left(3 \pi^{2}\right)\right) g_{s}^{2},
\end{aligned}
$$

with the numerical coefficients $c_{\lambda} \simeq 25 / 2-9 \pi /(2 \sqrt{ } 3) \simeq 4.3, c_{t} \simeq 6.1$. Thus

$$
\frac{\delta_{\lambda}}{\delta_{t}^{w}} \simeq \frac{c_{\lambda}}{c_{t}}\left(\frac{m_{H}}{m_{t}}\right)^{2} \simeq 0.3
$$

Then

$$
\rho_{t}=\rho_{t}^{0}\left(1+c_{1} \lambda-c_{2} g_{t}^{2}-c_{s} g_{s}^{2}\right) .
$$

The correction $\delta_{t}^{Q C D} \sim 5 \%$ is the most important one, acting to diminish slightly the ratio. Both corrections, $\delta_{t}^{w}, \delta_{\lambda}$, are of opposite sign and very small, of the order of $1 \%$.

\section{SM Renormalization group equations.}

We explore here the behaviour of the mass ratio $(3,31)$ at higher scales. We consider first a reduced system of one-loop renormalization group equations where only the Higgs self-coupling $\lambda$ and the top Yukawa coupling $g_{t}$ appear. All the other couplings are considered very small or not running at all. The RGE equations for the individual couplings take the form (see for example [6-9]) (with $t=\log (\mu / \Lambda)$, expression valid for high, but no so high, scales $\mu>>m_{t}, m_{H}$, or for $\Lambda \rightarrow \infty$ ):

$$
\begin{aligned}
\frac{d g_{t}^{2}}{d t} & =\frac{9}{16 \pi^{2}} g_{t}^{4}, \\
\frac{d \lambda}{d t} & =\frac{6}{16 \pi^{2}}\left(4 \lambda^{2}+2 \lambda g_{t}^{2}-g_{t}^{4}\right) .
\end{aligned}
$$

If we introduce the variable

$$
R=\frac{\lambda}{g_{t}^{2}},
$$

the RGE equations for $g_{t}, R$ and $\rho_{t}(t)$ become decoupled with nested solutions, $g_{t}=g_{t}(\mu), R=$ $R\left(g_{t}\right), \rho_{t}=\rho_{t}(R)$. In addition to Eq.(52), we have

$$
\begin{aligned}
g_{t}^{2} \frac{d R}{d g_{t}^{2}} & =\frac{1}{3} f(R), \\
\frac{d \rho_{t}}{d R} & =-\frac{3 \rho_{t}}{2 f(R)}\left(1+\frac{2 f(R)}{3 R}\right) .
\end{aligned}
$$


with $f(R)=8 R^{2}+R-2$. The equations $(52,55,56)$ can be solved explicitly, in particular for the ratio $\rho_{t}$ we can write

$$
\rho_{t}=k\left(\frac{R_{0}-R}{R_{1}+R}\right)^{R_{0}-R_{1}} R^{2},
$$

where $R_{0}, R_{1}$ are the fixed points of the equation (55), $f\left(R_{0,1}\right)=0$. For a light Higgs and large top mass the ratio $R$ is small, at low scales $R^{\exp } \sim 10^{-1}$, Eq.(36). For such a small $R$ the solution of the differential equations is approximately:

$$
R\left(g_{t}\right)=R_{c}-\frac{4}{3} \log g_{t},
$$

and

$$
\rho_{t} \sim k R^{2} \sim\left(R_{c}-\frac{4}{3} \log g_{t}\right)^{2} \sim k R_{c}^{2} \sim \rho_{t}^{0} .
$$

At large energies $\left(\mu>>m_{t}\right.$, as long as $R>0$ or $\left.\lambda>0\right)$, the ratio $\rho_{t}(\mu)$ keeps approximately constant, only sligtly decreasing with the logarithm of $g_{t}$.

If we consider a reduced Higgs-top-strong system where the $\lambda, g_{t}, g_{s}$ are non-vanishing and allowed to run together with the ratios $R, \rho_{t}$. One ends with a similar system of equations where the evolution of $\rho_{t}$ is of the type $g_{t}^{2} d \rho_{t} / g_{t}^{2} \sim \rho_{t} h\left(R, g_{t}^{2}\right)$ and similar results are obtained.

At higher energies, and for more quantitative results, a full treatment is needed. Present state-ofthe-art NLO and NNLo constraints on SM vacuum stability [12] seems to guarantee stability, and then a reasonably stable, positive, value for the quartic coupling, for a Higgs mass $m_{H} \sim 126 \mathrm{GeV} / \mathrm{c}^{2}$ and to very high scales.. If we assume a stable behaviour for $\lambda$ and ignoring the very modest variation of the coupling factor $g^{2}+g^{\prime 2}$,

$$
\rho_{t}(\mu) \sim \rho_{t}^{0} \frac{g_{t}(\mu)}{g_{t}^{0}} .
$$

the variation of the mass ratio $\rho_{t}$ is governed by the top Yukawa up to scales where new physics is expected to emerge.

\section{Conclusions and further discussion}

We expect new physics that cuts off the divergent top, gauge and higgs loop contributions to the Higgs Mass at scales $\lesssim 10 \mathrm{TeV}$. Many different possibilities have been well explored, they usually include, more or less ad-hoc, new particles with properties tightly associated to those of the SM. Some of these possibilities are for example (and any combinations among them)[10, 11]: a) The new particles are just the, softly broken, SUSY, superpartners with couplings and Yukawas strongly dictated by supersymmetry and the soft breaking itself. b) The Higgs is a composite resonance, or c) The "Little" Higgs is a pseudo-Nambu-Goldstone boson with respect a "softly" broken approximate global symmetry. This scalar sector is accompanied by some new particles belonging to enlarged multiplets together with the SM particles.

It is a general feature that, in all or most of these models, the quartic self coupling, and then the Higgs mass, is related to the gauge coupling constants and to the top yukawa in a more or less explicit way, reminding of the relation (36) suggested by the experimental evidence $\rho_{t} \simeq 1$. The reason is clear [10], the new one-loop which are proportional to the couplings of the SM gauge sector (or to a subsector of an enlarged gauge sector) have to match and cancel the top and the other cuadratic loops. 
We will briefly review the situation in the MSSM and Littlest Higgs scenarios. In the MSSM, the tree level top, gauge and lowest Higgs boson masses together their respective Yukawa couplings are given by the expressions

$$
\begin{aligned}
v^{2} & =v_{1}^{2}+v_{2}^{2}, \quad \tan \beta=v_{2} / v_{1}, \\
m_{W} & =g \frac{v}{2}, \quad m_{Z}=\sqrt{g^{2}+g^{\prime 2}} \frac{v}{2} \\
m_{t} & =g_{t} \frac{v}{2} \sin \beta .
\end{aligned}
$$

where the tree level Higgs quartic coupling is determined in terms of the gauge couplings

$$
\lambda^{0}=\left(g^{2}+g^{\prime 2}\right) \cos ^{2} 2 \beta .
$$

Quantum corrections to the Higgs quartic coupling are very important. They lead for an expression for the lower neutral Higgs mass, of the form [13]

$$
\begin{aligned}
m_{H}^{2} & =m_{Z}^{2} \cos ^{2} 2 \beta+\delta m_{H}^{2} \\
& =m_{Z}^{2} \cos ^{2} 2 \beta+f \frac{3 G_{F}}{\sqrt{2} \pi^{2}} m_{t}^{4}
\end{aligned}
$$

where the factor $f$ include logarithmic corrections, mainly associated to stops. From the expression (64) and from $m_{H}^{2}=2 \lambda(\mu) v^{2}$ we can extract an improved value for the quartic effective coupling

$$
\lambda(\mu)=\frac{m_{H}^{2}}{2 v^{2}}\left(1+\delta_{\lambda}(\mu)\right) .
$$

The effective quartic coupling is of the natural size $\lambda \sim o\left(g^{2}, g_{t}^{4}\right)$. In terms of these quantities the mass ratio $\rho_{t}$ is simply given by

$$
\begin{aligned}
\rho_{t}^{M S S M} & =\frac{m_{Z} m_{t}}{m_{H}^{2}}= \\
& =\frac{\sqrt{g^{2}+g^{\prime 2}} g_{t} \sin \beta}{\left(g^{2}+g^{\prime 2}\right) \cos ^{2} 2 \beta+g_{t}^{4} \sin ^{4} \beta 3 f / \pi^{2}} .
\end{aligned}
$$

In the context of the MSSM, the experimental evidence $\rho_{t} \simeq 1$ is a strong hint for the existence of a relation among the parameters of the expression above, principally top Yukawa and $\tan \beta$ together with the gauge couplings.

As a second illustrative example, let us mention the "Littlest" Higgs scenario [10]. Here the usual Higgs doublet, is the lightest of a set of pseudo goldstone bosons in an non-linear sigma model including in its gauge group different $S U(2) \times U(1)$ factors. The product group is broken to the diagonal, identified as the SM electroweak gauge group. The top Yukawa coupling generates a negative mass squared triggering electrowak symmetry breaking. New particles are added, in particular heavy top partners, which cancel the one loop quadratically divergent corrections. The quartic self coupling is related to the coupling constants of the gauge sector and to the top Yukawa with a natural size

$$
\lambda \sim o\left(g^{2}, g_{t}^{2}\right)
$$

reminding, for example, the expression (40) suggested by experimental evidence. Particular scenarios can be tuned so that either the gauge contributions or the top Yukawas dominate the Higgs quartic and $m_{H} \sim m_{Z}$ or $m_{H} \sim m_{t}$ as extreme cases. In fact we have seen, according to Eq.(3), that nature chooses, 
to a very high precision, just the geometric average of both extreme cases $m_{H}=\sqrt{m_{Z} m_{t}}$. It seems plausible that a Little Higgs scenario can be found where this value appears naturally. Approximate accidental global symmetries related to the Little Higgs scenario could play a role in the understanding of the $\rho_{t}$ ratio, as the global custodial $S U(2)_{c}$ symmetry [14] plays for the $\rho$ ratio.

In this short note we have presented some simple computations associated to the ratio of the product of $Z^{0}$ and top masses to the squared Higgs mass, $\rho_{t}$. We have shown how this ratio is suprisingly and robustly close to the unity at the $10^{-3}$ level. The Higgs mass seems to be just the geometrical mean of the $m_{Z}$ and $m_{t}$ masses.

We have briefly reviewed the theoretical predictions of this ratio in the SM and beyond. In the $\mathrm{SM}$, the Higgs selfcoupling $\lambda$ is undetermined. However, assuming that the value $\rho_{t}^{(\text {exp })} \simeq 1$ is not merely a coincidence, the relation Eq.(31) tell us that the scalar self-coupling and the spin 1 and spin $1 / 2$ top couplings are subject to the tree level equality

$$
\lambda \simeq c \sqrt{g^{2}+g^{\prime 2}} g_{t} \simeq c g g_{t}
$$

where $c$ is a numeric factor of order $\sim o(1)$. Such a relation is not very much affected by quantum effects at least up to scales $\mu \sim m_{t}$ or slightly higher.

Moreover, the combined use of both the relations $\rho_{t}^{(\exp )} \simeq 1, \rho_{W t}^{(\exp )} \simeq 1$ leads to the SM tree level relation (not very much affected by quantum effects)

$$
\lambda \simeq g^{2}+g^{\prime 2}
$$

In a model independent way, the relations $\rho_{t} \simeq \rho_{W t} \simeq 1$ imply that any two of the quantities $m_{H}, m_{W}, m_{Z}, m_{t}$ can be written in terms of the other two. Taking into account also the relation $\rho \simeq 1$ we can write any two mass ratios as a function of, exclusively, the Weinberg angle (with a precision of the order of $1 \%$ or better) $\frac{m_{i}}{m_{j}} \simeq f_{i j}\left(\theta_{W}\right), i, j=W, Z, H, t$. In the limit $\cos \theta_{W} \rightarrow 1$ all the masses would become equal $m_{Z}=m_{W}=m_{t}=m_{H}$. The existence of such relations could be interpreted as a hint for an instrumental role, together with some other unknown symmetry, of the $S U(2)_{c}$ custodial symmetry in the explanation of the $m_{H} / m_{t}$ ratio [14].

The closeness of the parameter $\rho_{t}, \rho_{W t}$ to one, "the Higgs mass coincidence problem", might be merely a coincidence or an artifact of the early status of the Higgs discovery, which will dissapear with any new measurement. It is tempting to think that such a value of the ratios are, on naturalness grounds, a signal of a deeper mechanism or symmetry.

\section{acknowledgments}

The author wish to thank to the ICFNP organizators for the participation invitation. This work has been supported in part by a grant from the Spanish Ministry of Science.

\section{References}

[1] E. Torrente-Lujan, Published in Eur.Phys.J. C74 (2014) 2744, [ arXiv:1209.0474 [hep-ph]] .

[2] J. Beringer et al. (Particle Data Group), Phys. Rev. D86, 010001 (2012) and 2013 partial update for the 2014 edition.

[3] G. Aad et al. [ATLAS Collaboration], Phys. Lett. B 716 (2012) 1 [arXiv:1207.7214 [hep-ex]]. G. Aad et al. [ATLAS Collaboration], Science 338 (2012) 1576. G. Aad et al. [ATLAS Collaboration], Phys. Lett. B 726 (2013) 120 [arXiv:1307.1432 [hep-ex]]. G. Aad et al. [ATLAS Collaboration], Phys. Lett. B 726 (2013) 88 [arXiv:1307.1427 [hep-ex]]. D. Orestano [ATLAS Collaboration], Int. J. Mod. Phys. D 22 (2013) 1330015. 
[4] S. Chatrchyan et al. [CMS Collaboration], Phys. Lett. B 716 (2012) 30 [arXiv:1207.7235 [hep-ex]]. S. Chatrchyan et al. [CMS Collaboration], Phys. Rev. Lett. 110 (2013) 081803 [arXiv:1212.6639 [hep-ex]].

[5] C. Wetterich, DESY-87-154. G. Altarelli and G. Isidori, Phys. Lett. B 337 (1994) 141. B. Pendleton and G. G. Ross, Phys. Lett. B 98 (1981) 291.

[6] M.E. Machacek and Vaughn, Nucl. Phys B222 (1983) 83; ibid. B236 (1984) 221; ibid.B249 (1985) 70; C. Ford, D.R.T. Jones, P.W. Stephenson and M.B. Einhorn, Nucl. Phys. B395 (1993) 17. K. Inoue, A. Kakuto and S. Takeshita, Prog. Theor. Phys. 67 (1982) 1889; ibid. 68 (1982) 927; S.P. Martin and M.T. Vaughn, Phys. Rev. D50 (1994) 2282. D. G. Cerdeno, E. Gabrielli, S. Khalil, C. Munoz, E. Torrente-Lujan Nucl. Phys. B 603 (2001) 231 [hep-ph/0102270]. R. Akers et al., Phys. Lett. B 327 (1994) 397.

[7] V. D. Barger, M. S. Berger and P. Ohmann, Phys. Rev. D 47 (1993) 1093 [hep-ph/9209232].

[8] B. Schrempp and M. Wimmer, Prog. Part. Nucl. Phys. 37 (1996) 1 [hep-ph/9606386].

[9] C. Wetterich, Proc. Trieste HEP Workshop (1987) p.403, and preprint DESY-87-154 (1987).

[10] N. Arkani-Hamed, A. G. Cohen, E. Katz and A. E. Nelson, "The Littlest Higgs," JHEP 0207 (2002) 034 [hep-ph/0206021]. M. Schmaltz and D. Tucker-Smith, "Little Higgs review," Ann. Rev. Nucl. Part. Sci. 55 (2005) 229 [hep-ph/0502182].

[11] A. Pomarol, CERN Yellow Report CERN-2012-001, 115-151 [arXiv:1202.1391 [hep-ph]]. B. Gripaios, A. Pomarol, F. Riva and J. Serra, JHEP 0904 (2009) 070 [arXiv:0902.1483 [hep$\mathrm{ph}]]$.

[12] L. A. Anchordoqui, I. Antoniadis, H. Goldberg, X. Huang, D. Lust, T. R. Taylor and B. Vlcek, arXiv:1208.2821 [hep-ph]. M. Lindner, M. Sher and H. W. Zaglauer, Phys. Lett. B228, 139 (1989). M. Sher, Phys. Rept. 179, 273 (1989). J. Ellis, J. R. Espinosa, G. F. Giudice, A. Hoecker and A. Riotto, Phys. Lett. B 679, 369(2009) [arXiv:0906.0954 [hep-ph]]. Z. -z. Xing, H. Zhang and S. Zhou, arXiv:1112.3112 [hep-ph]. L. J. Hall and Y. Nomura, JHEP 1003, 076 (2010) [arXiv:0910.2235 [hep-ph]].

[13] M. Drees, R. Godbole and P.Roy, Theory and phenomenology of sparticles: An account of four-dimensional $N=1$ supersymmetry in high energy physics. Hackensack Ed., World Scientific (2004).

[14] P. Sikivie, L. Susskind, M. B. Voloshin and V. I. Zakharov, Nucl. Phys. B 173, 189 (1980). A. Pomarol and R. Vega, Nucl. Phys. B 413, 3 (1994) [arXiv:hep-ph/9305272]. J. M. Gerard and M. Herquet, Phys. Rev. Lett. 98, 251802 (2007) [arXiv:hep-ph/0703051].

[15] E. Torrente-Lujan," The Higgs and top masses, the ratio $\rho_{t}$ and the $S U(2)_{c}$ custodial symmetry " (To appear). 